\title{
Blind Estimation of Multiple Digital Signals Transmitted over FIR Channels
}

\author{
Alle-Jan van der Veen, Shilpa Talwar and Arogyaswami Paulraj
}

\begin{abstract}
Using oversampling and the finite-alphabet property of digital communication signals, it is possible to blindly identify an FIR channel carrying a superposition of such signals, provided they have the same (known) period, and certain rank conditions on the data and channel matrices are satisfied. In particular, this technique allows to separate finite alphabet signals, remove intersymbol interference, and synchronize the signals. An algorithm is proposed and tested on simulated data.
\end{abstract}

\section{INTRODUCTION}

In the context of blind identification of channels carrying digital communication signals, a number of algorithms have been proposed to (A) estimate a single FIR channel carrying one signal. In one class of these algorithms, initiated by Tong, $\mathrm{Xu}$ and Kailath [1], the signal is recovered by oversampling the channel output (viz. a.o. [1-4]). In the context of array signal processing, another scenario which admits blind identification is the case where $(B) M$ antennas receive a superposition of $d \leq M$ synchronized finitealphabet (FA) input signals via memoryless channels. An algorithm to recover the signals was recently proposed by Talwar, Viberg and Paulraj [5].

In this communication, we show that the above two scenarios can be combined to (C) blindly identify multiple FIR channels carryingd unsynchronized digital FA input signals that have the same symbol rate and alphabet. See figure 1. The new identification algorithm combines algorithms for $(A)$ and $(B)$. In the context of array signal processing, the algorithm can be used to separate, synchronize and recover a number of incoming digital signals, arriving from different or possibly the same directions, and distorted by multipath with finite delay spread.

\section{DATA MODEL}

We describe a digital signal $s(t)$ as a sequence of dirac pulses, $s(t)=\sum_{-\infty}^{\infty} s_{k} \delta(t-k)$. For convenience, the symbol rate is normalized to $T=1$. An array of $M$ sensors, with outputs $x_{1}(t), \cdots, x_{M}(t)$, receives $d$ digital signals $s_{1}(t), \cdots, s_{d}(t)$ through independent channels $h_{i j}(t)$. Each impulse response $h_{i j}(t)$ is a convolution of the shaping filter of the $i$-th signal and the actual channel from the $i$-th input to $x_{j}(t)$, including any propagation delays. This data model is written compactly as $\mathbf{x}(t)=H(t) * \mathbf{s}(t)$,

A.J. van der Veen was with Information Systems Laboratory, Stanford University, Stanford, CA 94305. He is presently with the Department of Electrical Engineering, Delft University of Technology, Delft, The Netherlands. S. Talwar is with the Scientific Computing Program, Stanford University, Stanford, CA 94305. A. Paulraj is with Information Systems Laboratory, Stanford University, Stanford, CA 94305. This research was partly supported by ARPA, contract no. F49620-91-C-0086, monitored by the AFOSR. where

$$
\begin{aligned}
& \mathbf{x}(t)=\left[\begin{array}{c}
x_{1}(t) \\
\vdots \\
x_{M}(t)
\end{array}\right], H(t)=\left[\begin{array}{ccc}
h_{11}(t) & \cdots & h_{1 d}(t) \\
\vdots & & \vdots \\
h_{M 1}(t) & \cdots & h_{M d}(t)
\end{array}\right], \\
& \mathbf{s}(t)=\left[\begin{array}{c}
s_{1}(t) \\
\vdots \\
s_{d}(t)
\end{array}\right] .
\end{aligned}
$$

If we sample each $x_{i}(t)$ over $N$ symbol periods at a rate $P \in \mathbb{N}$, where $P$ is the oversampling factor, then we can construct a data matrix $X$ as

$$
\begin{aligned}
& X=\left[\begin{array}{lll}
\mathbf{x}_{0} & \cdots & \mathbf{x}_{N-1}
\end{array}\right]
\end{aligned}
$$

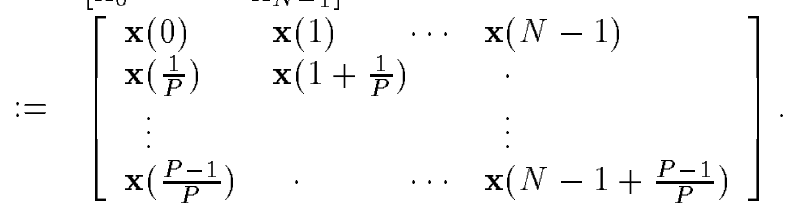

The $k$-th column $\mathbf{x}_{k}$ of $X$ contains the $M P$ samples taken in the $k$-th symbol period. If we assume that all $h_{i j}(t)$ are FIR filters of length at most $L \in \mathbb{N}: h_{i j}(t)=0, t \notin[0, L)$, then at most $L$ consecutive symbols of each signal play a role in $\mathbf{x}(t)$ at any given moment, and $X$ can be factored as

$$
X=H S_{T}
$$

where

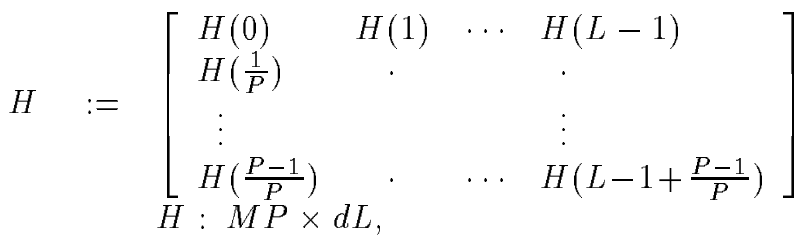

$$
\begin{aligned}
& S_{T}:=\left[\begin{array}{cccc}
\mathbf{s}_{0} & \ddots & \mathbf{s}_{N-2} & \mathbf{s}_{N-1} \\
\ddots & \ddots & \ddots & \mathbf{s}_{N-2} \\
\mathbf{s}_{-L+2} & \mathbf{s}_{-L+3} & \ddots & \ddots \\
\mathbf{s}_{-L+1} & \mathbf{s}_{-L+2} & \ddots & \mathbf{s}_{N-L}
\end{array}\right] \\
& S_{T}: d L \times N \text {, block-Toeplitz. }
\end{aligned}
$$

The blind identification problem is to estimate $H$ and $S_{T}$ from $X$. Note that for such a factorization to be unique, it is necessary that $H$ and $S_{T}$ have full column rank and row rank, respectively, which implies a.o. $M P>d L$. If this condition does not hold, we can extend $X$ to a block- 


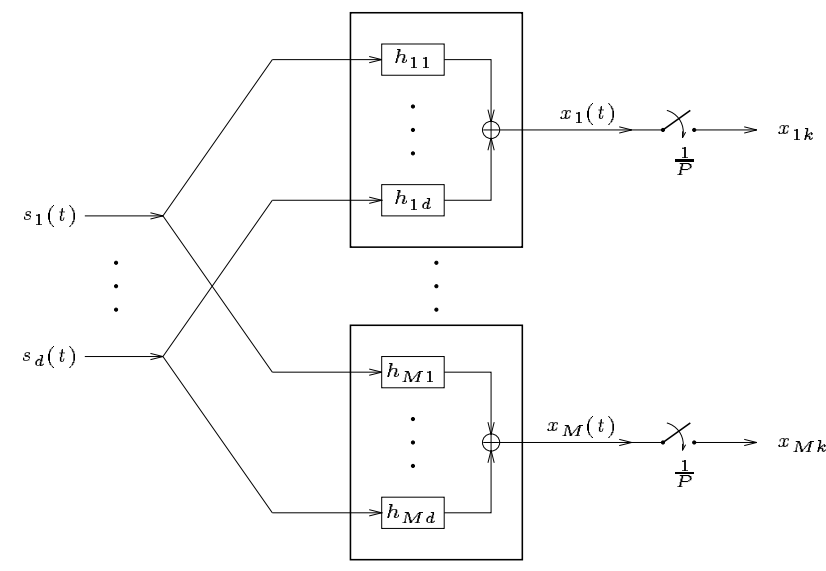

Fig. 1. Channel model. Input signals $s_{1}(t), \cdots, s_{d}(t)$ are synchronized dirac-pulse sequences.

Hankel matrix, by left-shifting and stacking $m$ times,

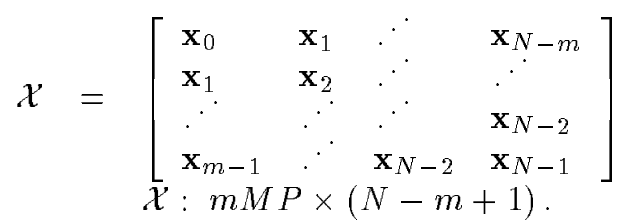

The augmented data matrix $\mathcal{X}$ has a factorization

$$
\begin{aligned}
& \mathcal{X}=\mathcal{H S}
\end{aligned}
$$

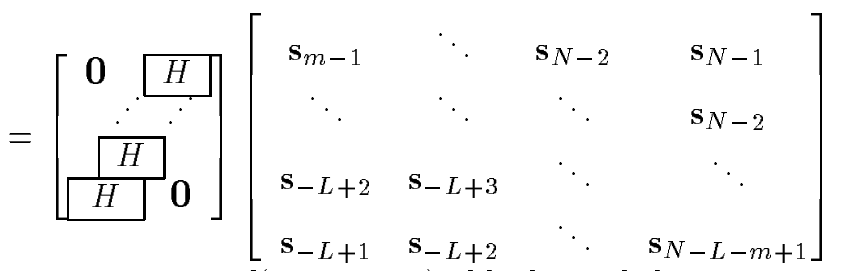

$$
\begin{aligned}
& \mathcal{H}: m M P \times d(L+m-1) \text { : block-Hankel, } \\
& \mathcal{S}: d(m+L-1) \times(N-m+1): \text { block-Toeplitz. }
\end{aligned}
$$

Now, necessary conditions for $\mathcal{X}$ to have a minimal-rank factorization $\mathcal{X}=\mathcal{H S}$ are that $\mathcal{H}$ is a 'tall' matrix and $\mathcal{S}$ is a 'wide' matrix, which for $L>1$ leads to

$$
\begin{aligned}
M P & >d \\
m & \geq \frac{d(L-1)}{M P-d} \\
N & >(d+1) m+d(L-1)-1
\end{aligned}
$$

Moreover, if $\mathcal{H}$ and $\mathcal{S}$ have full rank, then $\operatorname{row}(\mathcal{X})=\operatorname{row}(\mathcal{S})$ and $\operatorname{col}(\mathcal{X})=\operatorname{col}(\mathcal{H})$.

\section{BLIND IDENTIFICATION}

Our identification scheme is based on determining a factorization $\mathcal{X}=\mathcal{H S}$, such that either $\mathcal{S}$ is a blockToeplitz matrix with a specified row span, or $\mathcal{H}$ is a blockHankel matrix with a specified column span. However, such subspace information alone results in an ambiguity, since $\mathcal{X}=\left(\mathcal{H} D^{-1}\right)(D \mathcal{S})$ is a factorization with the same subspaces for $D=\operatorname{diag}[A, \cdots, A]$ and $A$ any invertible $d \times d$ matrix. This ambiguity is resolved in a second step, by taking advantage of the finite-alphabet property of the signals. In general, estimating $\mathcal{H}$ is computationally easier (for large $N$ ), but estimating $\mathcal{S}$ directly might be more accurate. In this letter, we focus on estimating $\mathcal{S}$.

\section{A. Computation of $\mathcal{S}$}

Let $G$ be a matrix whose columns constitute a basis for $\operatorname{ker}(\mathcal{X})$. If $\mathcal{H}$ has full column rank, then $G$ has dimensions $(N-m+1) \times(N-(d+1) m-d(L-1)+1)=: m_{G} \times N_{G}$. Moreover, $\mathcal{X} G=0 \Rightarrow \mathcal{S} G=0$. Using the fact that $\mathcal{S}$ is block-Toeplitz, we obtain

$$
\mathcal{S} G=0 \quad \Leftrightarrow \quad S G_{T}=0
$$

where

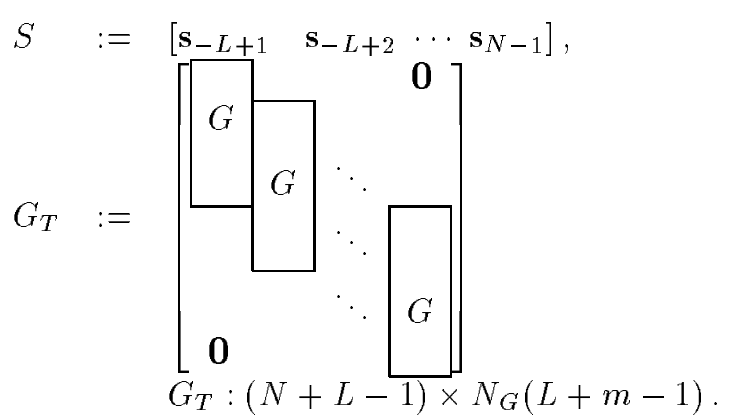

If $G_{T}$ is a wide matrix, then $\operatorname{ker}\left(G_{T}^{*}\right)$ is $d$-dimensional and determines $S$, but only up to a left invertible $d \times d$ matrix $A$. This is because $Y=A S$ also satisfies $Y G_{T}=0$. To identify $S$, we impose the finite alphabet structure on $S$ using the ILSP algorithm presented in [5]. ILSP is an iterative algorithm that solves the factorization $(Y=A S$ : $A, S$ full rank, $[S]_{i j} \in \mathcal{F} \mathcal{A}$ ), where $\mathcal{F} \mathcal{A}$ is a pre-specified finite alphabet. In its simplest formulation, the algorithm consists of alternating projections: starting, e.g., with $S^{(0)}=Y$, where in our case $Y$ is a matrix containing any basis for $\operatorname{ker}\left(G_{T}^{H}\right)$,

- Project $S^{(k)}$ onto $\operatorname{row}(Y): S^{(k) \prime}:=S^{(k)} Y^{\dagger} Y$,

- Project each $\left[S^{(k) \prime}\right]_{i j}$ onto the closest member of the alphabet, resulting in $S^{(k+1)}$.

The iteration generally converges very rapidly.

\section{B. Detection of $d$ and $L$}

If $\mathcal{H}$ and $\mathcal{S}$ have full column rank and row rank, respectively, then the rank of $\mathcal{X}$ is $d_{X}:=d(m+L-1)$. In principle, $d$ can be estimated by increasing $m$ by one, and looking at the increase in rank of $\mathcal{X}$. This property provides a useful detection mechanism even if the noise level is quite high since it is independent of the actual (observable) channel length $\hat{L}$. Furthermore, it still holds if all channels do not have equal lengths. If they do, then $L$ can be estimated from the estimated rank of $\mathcal{X}, \hat{d}_{X}$, and the estimated number of signals, $\hat{d}$, by $\hat{L}=\hat{d}_{X} / \hat{d}-m+1$.

\section{Remarks}

If the channels do not have equal lengths, then $\mathcal{H}$ is not full rank and the above algorithm has to be modified. Omitting the details, we mention that it is sufficient to set 
$L$ equal to the minimal channel length. This reduces the number of shifts of $G$ in (2), so that $\operatorname{ker}\left(G_{T}^{*}\right)$ is larger than before. Besides the symbol sequences, this space now also contains a number of shifted copies of them. After processing this larger space by ILSP, it is straightforward to detect the shifted copies and remove them.

\section{SIMULATION RESULTS}

In a simulation of a multiray wireless channel, we consider $d=2$ unsynchronized BPSK signals, each modulated by a raised-cosine shaping filter (modulation parameter 0.35 , and truncated to a length of 6 symbol periods). The signals are received by $M=2$ antennas spaced by half a wavelength. The simulated channel consists of four paths per signal, where each path is specified by an angle-ofarrival $\alpha$, delay $\tau$, and complex damping factor $p$ :

$$
\begin{array}{rr}
s_{1}: & \left(\alpha_{i}, \tau_{i}, p_{i}\right) \in\left\{\left(-10^{\circ}, 0,1 e^{-2.72 j}\right),\left(-2^{\circ}, .3, .8 e^{.61 j}\right),\right. \\
\left.\left(-120^{\circ}, 1.2, .4 e^{.93 j}\right),\left(160^{\circ}, 2.1, .4 e^{1.64 j}\right)\right\} \\
s_{2}: \quad\left(\alpha_{i}, \tau_{i}, p_{i}\right) \in\left\{\left(10^{\circ}, .5,1 e^{-3.07 j}\right),\left(15^{\circ}, .9, .9 e^{1.17 j}\right),\right. \\
\left.\left(-40^{\circ}, 1.5, .5 e^{-1.06 j}\right),\left(150^{\circ}, 2.8, .3 e^{1.83 j}\right)\right\} .
\end{array}
$$

The resulting channel length is $L=9$. We took $N=50$ sampling intervals, with $P=5$ times oversampling, and set the SNR to $10 \mathrm{~dB}$ per signal.

The singular values of the resulting data matrix $\mathcal{X}$ are plotted in figure $2(a)$, for blocking factors $m=2,3,4$. The numerical rank of $\mathcal{X}$ is about $\hat{d}_{X}=10,12,14$, respectively, so that the number of signals is detected as $\hat{d}=2$ and the observed channel length is $\hat{L}=4$, rather than 9 . Figure $2(b)$ shows the singular values of $G_{T}$, for $m=3, \hat{d}_{X}=12$, and $\hat{L}=3,4$. For $\hat{L}=4$, the number of small singular values is indeed equal to $\hat{d}=2$; for $\hat{L}=3$, we also obtain two shifted copies of the two signals, as expected. For the choice ( $\hat{L}=3, \hat{d}=4)$, all bits were estimated correctly, and the estimated symbol standard deviations (before classification as +1 or -1 ) was about 0.20 . For $\hat{L}=4$, however, not all bits were estimated correctly, and the standard deviations were about 0.60 . In general, underestimating $L$ and overestimating $d$ yields significantly more accurate results. This is because ILSP now gets a larger responsibility in separating both the signals and their echos, which is favorable since the finite alphabet property is quite powerful. At this noise level, the alternative algorithm which first identifies $\mathcal{H}$ and then estimates $\mathcal{S}=\mathcal{H}^{\dagger} \mathcal{X}$ gave bit errors of about $15 \%$. More details are provided in [6].
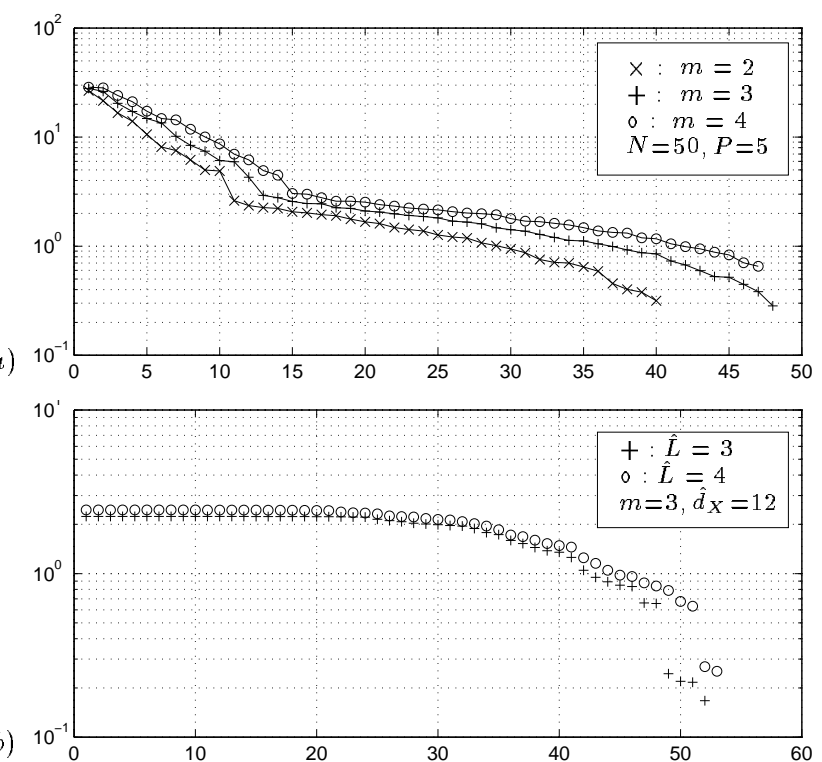

Fig. 2. (a) Singular values of $\mathcal{X}$, for different blocking factors $m$. (b) Singular values of $G_{T}$.

\section{REFERENCES}

[1] L. Tong, G. Xu, and T. Kailath, "A new approach to blind identification and equalization of multipath channels," in 25-th Asilomar Conf. on Signals, Systems and Computers, pp. 856860 vol. 2,1991

[2] H. Liu, G. Xu, and L. Tong, "A deterministic approach to blind equalization," in 27-th Asilomar Conf. on Signals, Systems and Computers, 1993.

[3] D.T.M. Slock, "Blind fractionally-spaced equalization, perfectreconstruction filter banks and multichannel linear prediction," in Proc. IEEE ICASSP, pp. IV:585-588, 1994.

[4] E. Moulines, P. Duhamel, J. Cardoso, and S. Mayrargue, "Subspace methods for the blind identification of multichannel FIR filters," in Proc. IEEE ICASSP, pp. IV:573-576, 1994.

[5] S. Talwar, M. Viberg, and A. Paulraj, "Blind estimation of multiple co-channel digital signals using an antenna array," IEEE Signal Processing Letters, vol. 1, pp. 29-31, Feb. 1994.

[6] A.J. van der Veen, S. Talwar, and A. Paulraj, "Blind identification of FIR channels carrying multiple finite alphabet signals," to appear in IEEE ICASSP-95, May 1995. 